\title{
Towards a direct band gap group IV Ge-based material
}

\author{
Tuan T. Tran ${ }^{\mathrm{a}, \mathrm{b}, *}$, Jay Mathews ${ }^{\mathrm{c}}$, J.S. Williams ${ }^{\mathrm{a}}$ \\ a Department of Electronic Materials Engineering, Research School of Physics and Engineering, Australian National University, Canberra, ACT 0200, Australia \\ ${ }^{\mathrm{b}}$ Department of Physics and Astronomy, Uppsala University, Box 516, S-751 20 Uppsala, Sweden \\ ${ }^{\mathrm{c}}$ Department of Physics, University of Dayton, Dayton, $\mathrm{OH} 45469$, USA
}

\section{A B S T R A C T}

A silicon-compatible laser source is of utmost importance for a successful photonic integrated circuit. The conventional solution using direct band gap III-V materials adds significant complexity into the fabrication process because the active materials have to be bonded or grown on a largely mismatched silicon substrate. Recently, germanium has been considered a promising material for silicon photonic applications due to its interesting electronic band structure. Several concepts to realise a direct band gap Ge-based material will be reviewed in this paper, such as: tensile strain combined with high n-type doping, high tensile strain created by micromachining, synthesis of Ge-Sn alloys by chemical vapour deposition and, in particular, synthesis of Ge-Sn alloys by ion implantation followed by pulsed laser melting (PLM). Besides providing a very high level of reproducibility and purity in conventional device fabrication, ion implantation followed by PLM is shown to have potential for realising an intrinsically direct band gap material of high quality. Producing a 10at. \%Sn alloy is now possible and a highly strain-relaxed layer can also be realised by this technique.

\section{Demand for a silicon-compatible laser}

Group IV semiconductor alloys, the Si-Ge-Sn system, are gaining significant interest as a key material towards realising a fully photonic integrated circuit and system-on-chip devices. Due to the downscaling of field effect transistors (FETs), the dimensions of the interconnects have to be reduced towards the limit where the delay caused by the interconnect is larger than by the gate. As a potential solution, interconnection using photons via a transparent medium is preferred to conventional electronic conduction via a metallic medium. This optical connection is believed to offer high bandwidth, low attenuation, low volume and the speed of light. Several all-silicon-based components for such a photonic integrated circuit (PIC) are almost fully developed, such as waveguides, modulators, photodetectors, resonators, and photonic crystals. The missing piece of the picture for a complete silicon PIC is a laser emitter as an efficient photon source.

Many attempts have been made throughout the years to integrate a laser source into a silicon circuit. The most effective method so far is a hybrid approach in which an efficient photon-emitting material, such as indium phosphide or gallium arsenide, is incorporated into silicon by wafer bonding $[1,2]$ or direct deposition of the III-V materials $[3,4]$. However, both of these methods considerably increase the complexity of the fabrication process. Another interesting option for monolithic integration of the optical amplifier is the use of the Raman scattering effect in silicon. The Raman gain coefficient in crystalline silicon was measured to be 4 orders of magnitude higher than in amorphous glass fibre, which can enable light amplification within a silicon waveguide [5]. Research on Raman silicon lasers has achieved some noticeable milestones [6-8]. Nevertheless, an electrically-driven Raman laser has not been demonstrated. Currently, light amplification of the Raman silicon laser is made by optical pumping with another laser source, which is not favourable for the photonic integration [9]. An additional potential material, that can be used as a laser source in a silicon photonic chip, is erbium (Er) doped silica. Erbium is a common dopant in optical applications due to its optical gain at the telecommunication wavelength of $1.55 \mu \mathrm{m}$. Er atoms can be introduced precisely by ion implantation into silica, which is a compatible material in silicon technology [10]. Optically-pumped lasing using Er-doped silica has been demonstrated [11]. A conceptual structure for an electricallydriven laser source has been also presented [12], in which an Er-doped silica layer of $60 \mathrm{~nm}$ thick is sandwiched between two p-type Si layers (Fig. 1). Carrier transport through the active region is of FowlerNordheim tunnelling between the $\mathrm{Si} / \mathrm{SiO}_{2}$ interface. According to the simulation, electroluminescence is possible in this structure, however, so far that possibility has not been demonstrated. An additional potential issue with Er-doped silica is that the luminescence depends on the concentration of Er atoms in the host material, which is limited to $1 \cdot 10^{22} \mathrm{~cm}^{-3}$ due to clustering. In other words, Er-doped silica is not an intrinsically lasing material and the lasing efficiency is inherently suppressed by the relatively low density of the active material in the host.

\footnotetext{
* Corresponding author.

E-mail address: tuan.tran@anu.edu.au (T.T. Tran).
} 


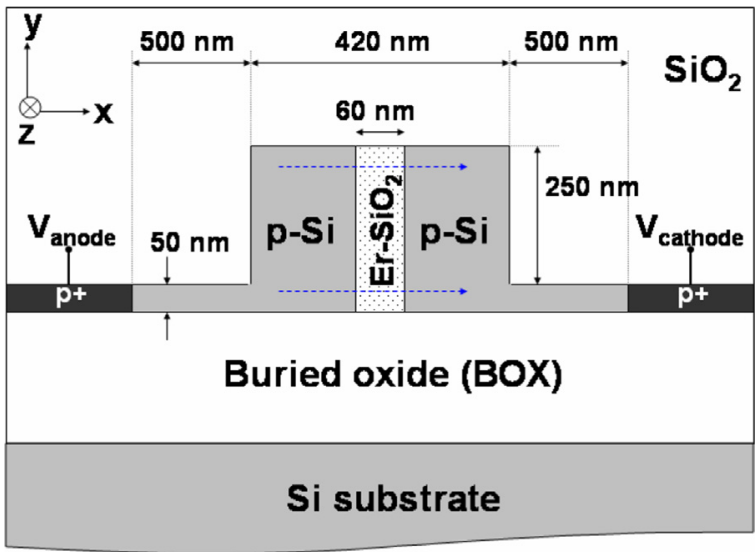

Fig. 1. A conceptual electrically-driven Er-doped silica laser [12].

\section{Ge-based materials for silicon photonic integrated circuits}

Within the last 10 years, great enthusiasm in the research and industrial communities has re-emerged for germanium (Ge) as an advanced material for future electronic and photonic applications. Similar to $\mathrm{Si}, \mathrm{Ge}$ is a group IV element of the periodic table. Both elements have some characteristics in common, such as the diamond cubic crystal structure and closely-related chemical properties, which make Ge highly compatible with current Si technology. For some criteria, Ge is even preferred, such as higher carrier mobility and a more favourable electronic band structure. At room temperature, the electron mobility of Ge is $\sim 3900 \mathrm{~cm}^{2} /(\mathrm{Vs}), 2.5$ times higher than that of Si. Its hole mobility is $\sim 1900 \mathrm{~cm}^{2} /(\mathrm{Vs})$, one of the highest mobilities of all types of semiconductors [13]. High carrier mobility is of the utmost importance for fast processing speeds of electronic devices. In terms of the band structure, Ge has a significantly smaller bandgap of $0.66 \mathrm{eV}$ as compared to $\mathrm{Si}(1.12 \mathrm{eV})$, which is beneficial for the current era of low power electronic devices. Smaller bandgap also gives rise to a broader absorption spectrum for Ge, at least up to $1.55 \mu \mathrm{m}$, valuable for some optoelectronic applications, such as photodetectors. In addition, Ge has another special property in its electronic band structure that could potentially place the material at the forefront of photonic integrated applications, as shown below.

Fig. 2 shows the calculated band structure of $\mathrm{Si}, \mathrm{Ge}$ and another group IV element with the diamond cubic crystal structure, $\alpha-S n$. In Si, the lowest conduction band is at the $\mathrm{X}$ valley, which is $\sim 2 \mathrm{eV}$ lower than the band gap at the $\Gamma$ valley, where the highest point of the valence band is situated. This makes Si an indirect bandgap material. Although
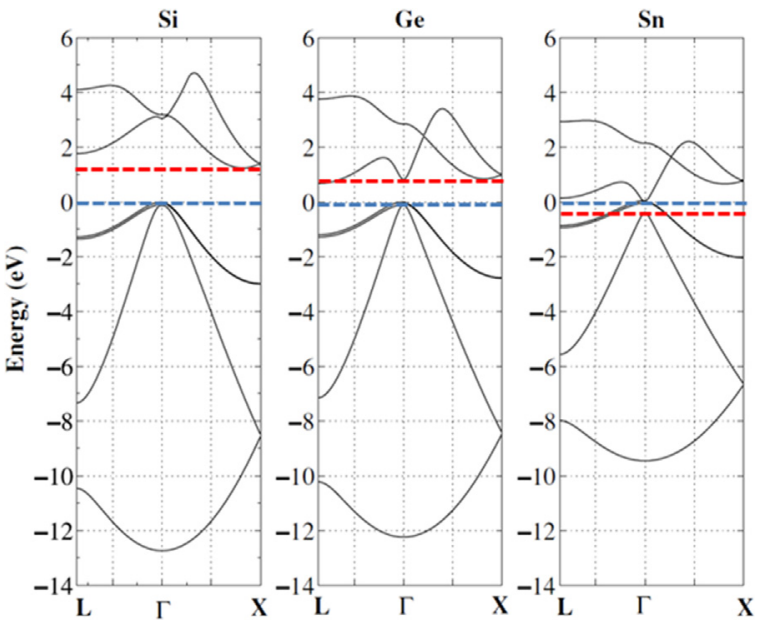

Fig. 2. Calculated band structure of bulk Si, Ge and $\alpha$-Sn [14].
$\mathrm{Ge}$ is also an indirect bandgap material with the bandgap at the $\mathrm{L}$ valley of $0.66 \mathrm{eV}$, the gap difference at the L valley and the $\Gamma$ valley is relatively small, of the order of $0.14 \mathrm{eV}$. Theoretical and experimental studies have shown that it is possible to make the bandgap at the $\Gamma$ valley smaller than at the L valley. In other words, proper band engineering can tune $\mathrm{Ge}$ into an intrinsically direct bandgap material.

Since the $70 \mathrm{~s}$, several groups have studied the effect of mechanical stress on the band structure of semiconductors, such as $\mathrm{Si}, \mathrm{Ge}$ and $\alpha$-Sn [15-18]. These studies showed that mechanical stress is more effective on states with lower effective mass because the deformation potential is inversely proportional to the state effective mass. The effective mass of the $\Gamma, \mathrm{L}$ and $\mathrm{X}$ valleys of the conduction band in $\mathrm{Ge}$ is $0.04,0.22$ and 0.34 , respectively; hence, the strength of the deformation potential on the $\Gamma$ valley is the largest [18]. Specifically, a biaxial tensile strain can move the $\Gamma$-valley downward faster than the L-valley. When the in-

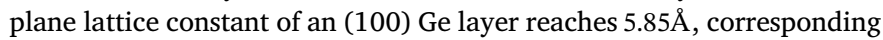
to $\sim 3.5 \%$ tensile strain, Ge is believed to be a direct bandgap material. For $\mathrm{Si}$, the amount of the required strain would be significantly higher, $10-13 \%$, and hence not practically achievable.

To enhance the optical transition at the $\Gamma$ valley in $\mathrm{Ge}$, the group of Kimerling et al. have proposed a concept combining tensile strain and high n-type doping as shown in Fig. 3 [19]. Although it is not sufficient, the tensile strain reduces the band gap difference at the $\Gamma$ and the $L$ valleys. The remaining difference is compensated by the high density of electrons in the conduction band to the extent that electrons begins to accumulate at the $\Gamma$ valley [19]. According to the calculation, a tensile strain of $0.25 \%$ combined with an electron density of $7.6 \cdot 10^{19} \mathrm{~cm}^{-3}$ would be sufficient for the purpose. The first experimental evidence of lasing using this concept has been reported by the same group in 2009 [20]. Tensile strain in Ge was achieved as a result of the large mismatch between the thermal expansion coefficients of $\mathrm{Ge}\left(8.51 \cdot 10^{-6} \mathrm{~K}^{-1}\right)$ and $\mathrm{Si}$ $\left(4.21 \cdot 10^{-6} \mathrm{~K}^{-1}\right)$ [21]. A Ge layer grown on $\mathrm{Si}$ was annealed by rapid thermal annealing at $780^{\circ} \mathrm{C}$ for $30 \mathrm{~s}$, and, upon cooling, tensile strain accumulates in the Ge layer. Optical gain at a wavelength of $1.6 \mu \mathrm{m}$ was observed in the material by both optical and electrical pumping experiments [20,22-24]. However, a significant issue of this method is the absorption of the concentrated free carriers as well as the limited amount of electrically activated dopants that can be introduced into Ge. The free carrier absorption effect drastically increases the lasing threshold of the material. In addition, the solid solubility of the conventional n-type dopant in Ge, antimony (Sb), is limited to $\sim 1 \cdot 10^{19} \mathrm{~cm}^{-3}$ [25].

As a result of the above limitations, another concept that relies on pure mechanical strain was proposed. Very large tensile strain in the material is created via the process used to fabricate the micro-electromechanical systems (MEMS) [26-28]. The starting substrate is Ge-on-Si or Ge-on- $\mathrm{SiO}_{2}$ in which the Ge layer was initially strained to about $0.15 \%$, similar to the experiment of tensile strain combined with n-type doping. A process sequence involving lithography, dry etching and wet etching was used to create a suspended micro-bridge between two larger pads as shown in Fig. 4. The existing tensile strain in the Ge layer is transferred into a very high strain in the micro-bridge due to the

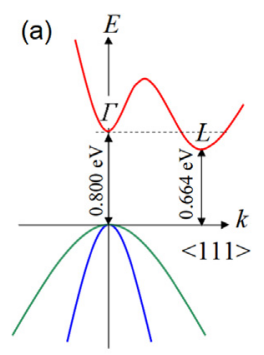

bulk Ge

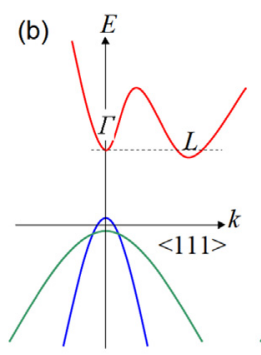

tensile strained $\mathrm{i}-\mathrm{Ge}$

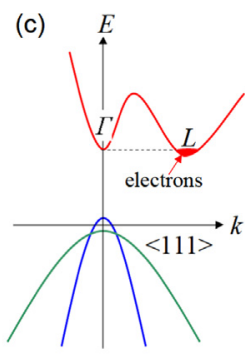

tensile strained $\mathrm{n}^{+} \mathrm{Ge}$
Fig. 3. Schematic band structure of pristine Ge (a), partially tensile-strained Ge (b) and tensile-strained Ge with high n-type doping (c) [19]. 

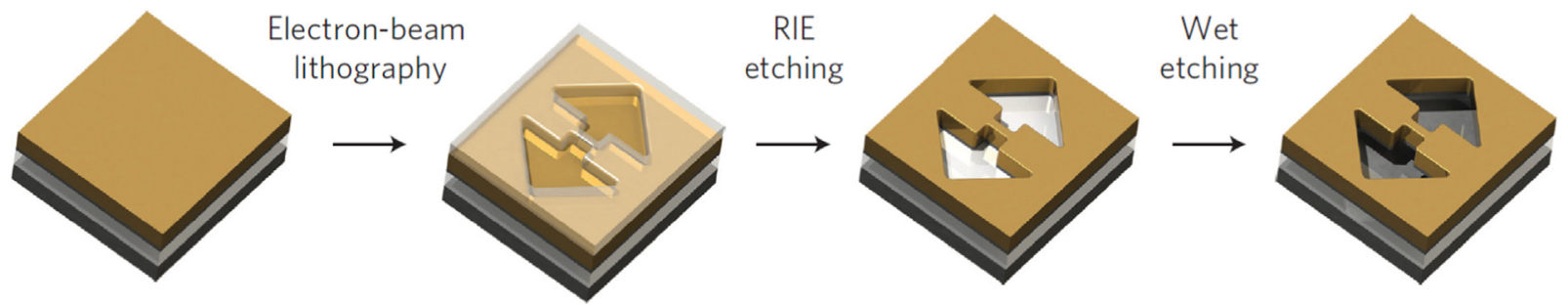

Fig. 4. The MEMS process to induce large tensile strain in the Ge micro-bridge [26].

much smaller cross-sectional area of the bridge. Consequently, a uniaxial tensile strain of $3.1 \%$ has been achieved. Although the direct bandgap transition has not been surpassed in this experiment, photoluminescence (PL) and Raman spectroscopy showed a red shift of $0.21 \mathrm{eV}$ compared to the bulk material, and a strong increase of integrated PL intensity [26].

\section{Ge-Sn alloy as an intrinsically direct band gap material}

The tensile strain concept in the previous section successfully demonstrated the possibility of a direct band gap transition in Ge, although an intrinsically direct bandgap material has not yet been realised by this method. The gain properties of the device relied either on the very high n-type doping or on a very large tensile strain created by micro-machining, both of which considerably prevent the material from practical applications in photonic integrated circuits. For the remaining part of this review, the alloy of Ge and $\alpha$-Sn will be presented as a potential solution for a group IV laser application. With an appropriate concentration of $\mathrm{Sn}$ and inherent strain, the alloy can become an intrinsically direct band gap semiconductor.

Theoretical studies on the band structure of Ge, $\alpha$-Sn and the combined band structure of the Ge-Sn alloy have been carried out [14,29-32]. Initially, it was calculated that at least 20at. \%Sn is required for the indirect-to-direct band gap crossover [30,31]. Simple linear calculations using Vegard's law gives the crossover Sn concentration at $\sim 22 a t . \%$. Later more realistic calculations reveal a significantly lower $\mathrm{Sn}$ concentration for the indirect-direct transition, such as $6.3 a t . \%$ [32] and 6.5at.\% [29], which is in better agreement with a large number of experimental data. The large discrepancy between the initial and the more recent studies is due to a larger-than-expected bowing parameter [33]. Fig. 5 from Ref. [29] shows the correlation between the band gap of the Ge-Sn alloy and the Sn concentration as well as the in-plane biaxial strain in the material. Two important points to be noticed from Fig. 5 are: 6.5 at. \% is the required Sn concentration for a fully relaxed alloy to have a direct band gap and a higher Sn concentration is necessary for a compressively strained alloy.

Unlike the Si-Ge alloy in which the two elements are miscible over the whole composition range, synthesis of Ge-Sn appears to be challenging due to a low solubility of $\mathrm{Sn}$ in Ge under equilibrium conditions. At room temperature, only $\sim 0.5 a t . \% S n$ is soluble in Ge. Above this concentration Sn clusters into another form of Sn, tetragonal $\beta$-Sn [34]. For this reason, synthesis of this alloy must employ non-equilibrium methods. In 1983, Oguz et al. fabricated the first Ge-Sn alloys by sputtering amorphous GeSn films, which were then subjected to UV pulsed laser annealing to induce crystallization [35]. The result was a microcrystalline structure rather than a single crystal. Within a few years, several groups were producing both amorphous and microcrystalline Ge-Sn films [36-38], but a single crystal film had yet to be realised. These early attempts at Ge-Sn growth also helped spur new theoretical studies on the band structure of Ge-Sn alloys [30,31], which in turn drove further efforts to produce single crystal Ge-Sn.

A number of non-equilibrium techniques have been applied to the Ge-Sn growth problem. Among suitable fabrication techniques, molecular beam epitaxy (MBE) and chemical vapour deposition (CVD) have

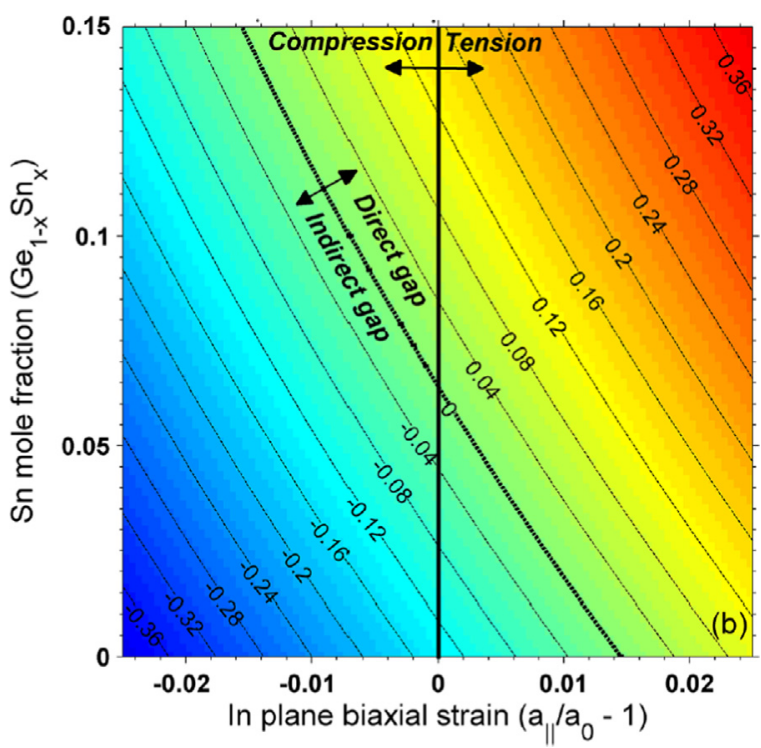

Fig. 5. The dependence of the indirect-direct bandgap transition on the Sn concentration and the in-plane biaxial strain [29].

been favoured over the past two decades. Early MBE studies successfully produced thin, single crystal Ge-Sn films, but the thicknesses were limited to only a few nanometres [39,40]. A major problem encountered during growth was the tendency of Sn to segregate toward the surface of the film. This could only be countered by lowering the growth temperature to $\sim 100^{\circ} \mathrm{C}$, placing severe limits on the critical thicknesses of the films. In 1997, He and Atwater pioneered the use of MBE for the synthesis of the Ge-Sn alloys. Their breakthrough solution was the use of a low energy ion beam of an inert element $\mathrm{Ar}^{+}$ $(30-100 \mathrm{eV})$ to act as a collisional mixer to prevent surface segregation of the Sn atoms. The energy of the ion beam was very low in order not to create significant ion beam damage in the material, but sufficient to promote the incorporation of $\mathrm{Sn}$ in the layer [41]. Optical absorption measurements revealed that the direct band gap transition lies from $6 a t$. \% to 11at. \% of Sn, which was much lower than the values predicted at that time $[30,31]$. However, the quality of the material was not sufficiently good to utilise this material for devices. Other reports of successful experiments with MBE can be found in Ref. [42-45].

CVD is a popular growth method, but in the 1990s it was not a feasible method for Ge-Sn growth due to the lack of a stable precursor for Sn. The energy of the Sn-H bond $(251 \mathrm{~kJ} / \mathrm{mol})$ is significantly lower than that of Si-H $(318 \mathrm{~kJ} / \mathrm{mol})$ and Ge-H $(288 \mathrm{~kJ} / \mathrm{mol})$, so stannane, which is the $\mathrm{Sn}$ analogue of silane or germane, would not be suitable as a precursor. The group of Kouvetakis and Menendez pioneered the use of CVD as a viable method for Ge-Sn alloy synthesis. In 2001, Taraci et al. proposed a new route to GeSn alloys via chemical vapour deposition using highly reactive Sn-containing gaseous precursors [46]. By using deuterium (2D or D) to increase the kinetic stability of the compound, viable precursors were achieved. The initial film in this study was grown on $\mathrm{Ge}$ using the precursors $\mathrm{Ge}_{2} \mathrm{H}_{6}$ and $(\mathrm{Ph}) \mathrm{SnD}_{3}\left(\mathrm{Ph}=\mathrm{C}_{6} \mathrm{H}_{5}\right.$ (phenyl), and $\mathrm{D}=$ deuterium), had a thickness of $55 \mathrm{~nm}$ and appeared to 
have a diamond cubic structure with reasonable quality. A short time later, Bauer et al. took this idea further and demonstrated the growth of epitaxially stabilized GeSn films directly on $\mathrm{Si}(100)$ substrates using $\mathrm{Ge}_{2} \mathrm{H}_{6}$ and the custom precursor deuterated stannane $\mathrm{SnD}_{4}[47,48]$. This latest precursor became the basis for CVD growth of Ge-Sn and it was used to fabricate the first Ge-Sn alloys with Sn content up to a few percent and thicknesses of $500 \mathrm{~nm}$ or greater.

By 2009, the crystal quality of alloy films grown using CVD with $\mathrm{SnD}_{4}$ was significantly improved, which lead to a number of significant developments for devices based on Ge-Sn. Mathews et al. demonstrated the first photodetectors made from Ge-Sn alloys, and detectors with $2 \%$ Sn were shown to cover the entire telecommunications spectrum [49]. Since then, photodetectors with Sn content up to $16 \%$ have been achieved using this method $[50,51]$. The CVD approach using $\mathrm{SnD}_{4}$ also lead to the first observable luminescence from Ge-Sn alloys. Photoluminescence with tunable emission wavelength was demonstrated in Ge-Sn films [52], and the first light-emitting diodes were produced [53].

While the use of deuterated stannane lead to the fabrication of high quality Ge-Sn films, adoption of this technique has been very limited. The production of the precursor gas is somewhat complicated, and $\mathrm{SnD}_{4}$ is a volatile compound at room temperature, so it must be stored at cryogenic temperatures. These conditions have prevented the widespread use of this method, and this has prompted research into finding others-based precursor gases. An alternative chemical route for a stable Sn precursor in CVD has been the use of commercially available tin chloride $\left(\mathrm{SnCl}_{4}\right)$, initially proposed by Vincent et al. [54]. $\mathrm{SnCl}_{4}$ is a stable liquid commonly used in the glass industry for Sn coating. For the CVD process, the liquid can be vaporised into the reaction chamber by a vapour station. The first film successfully grown with $\mathrm{SnCl}_{4}$ was reported to be defect-free, $40 \mathrm{~nm}$ thick, with a Sn concentration of $8 a t . \%$ [54]. The film was also shown to be stable under annealing at $500^{\circ} \mathrm{C}$ for 30min. This promising solution has been adopted by several other groups who have reported a large number of successful studies using $\mathrm{SnCl}_{4}$ [55-57]. Among those, Wirths et al. published the first lasing results from a Ge-Sn alloy in 2015 (Fig. 6) [58]. The lasing experiment was conducted with a CVD-grown 560nm thick partially-relaxed Ge-Sn layer on Ge, having a Sn concentration of $\sim 12.6$ at. \%. Lasing was observed up to a temperature of $90 \mathrm{~K}$ and at $20 \mathrm{~K}$ the lasing threshold was $325 \mathrm{~kW} / \mathrm{cm}^{2}$. In 2016, an improved lasing result was reported by another group, in which lasing was observed up to $110 \mathrm{~K}$ and the minimum lasing power was $68 \mathrm{~kW} / \mathrm{cm}^{2}$ at the temperature of $10 \mathrm{~K}$ [59]. Shortly later, by the same group was the lasing operation observed up to the temperature of $180 \mathrm{~K}$ [60]. These results demonstrate that the Ge-Sn alloy is progressing rapidly as a promising material for group IV-based

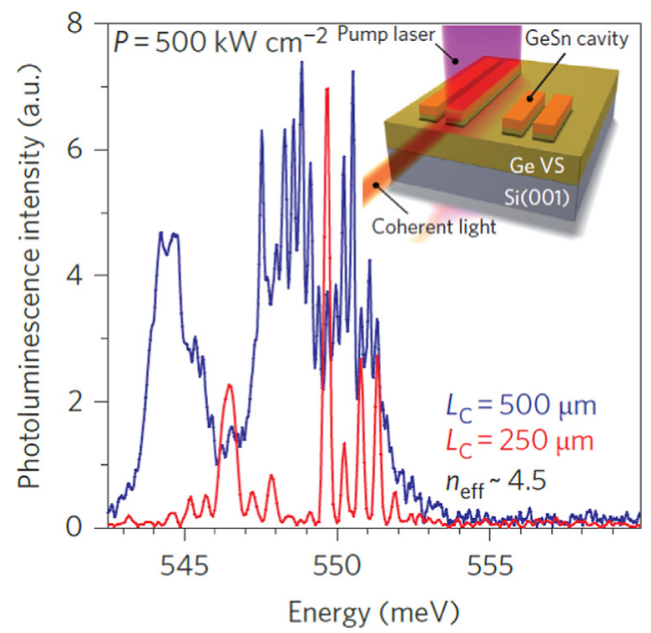

Fig. 6. Lasing evidence observed in the $250-500 \mu \mathrm{m}$ waveguide structures fabricated from a Ge-Sn alloy [58]. integrated photonics applications.

To date CVD is perhaps the most successful method for synthesising the Ge-Sn alloy. The technique is also somewhat industrially relevant. However, the use of the aforementioned $\mathrm{Sn}$ precursors, $\mathrm{SnD}_{4}$ or $\mathrm{SnCl}_{4}$, does not come without some potential issues, such as the instability of $\mathrm{SnD}_{4}$ and the undesirable chlorine contamination related to the $\mathrm{SnCl}_{4}$, as indicated below. Although reports on the instability of $\mathrm{SnD}_{4}$ are very rare, it is known to have a very short lifetime at room temperature. For example, Spohn et al. showed that a very diluted $\mathrm{SnD}_{4}(1 \%$ in hydrogen) at a pressure of $45 \mathrm{psi}$, stored in an aluminium cylinder, has a lifetime of about 6 days at room temperature [61]. A normal storing condition of silane $\left(\mathrm{SiH}_{4}\right)$ is $\sim 100 \% \mathrm{SiH}_{4}$ at the pressure of 1100psi. Special treatment to the chemical container, such as OTS (octadecyltrichlorosilane) or Quercetin can increase the lifetime to 234days and 255days, respectively. However, at slightly higher $\mathrm{SnD}_{4}$ concentration and pressure, such as $4 \%$ and 150 psi, the lifetime reduces to about 5 days. To improve the lifetime, the chemical has to be stored at liquid nitrogen temperature, $(77 \mathrm{~K}) . \mathrm{SnD}_{4}$ is also toxic and flammable (NFPA 704 rating: health 4/4, fire 4/4) [62]. Regarding the use of the more stable Sn precursor, $\mathrm{SnCl}_{4}$, there is concern on the contamination of chlorine into the film during growth [63]. Products in the reaction chamber include chlorine and hydrochloric acid, which can act as an etchants or intrude into the film. However, there is so far no report of such contamination in literature.

\section{Ion beam synthesis of Ge-Sn alloys}

Ion implantation has long been accepted as a powerful method to precisely introduce foreign elements into a host material to modify the material properties, such as electrical, optical, mechanical and chemical. Besides being a very robust method, ion implantation is very flexible in the source materials for the implanted species. Hence, it is not subject to the sometimes uncertain chemistry of precursors. The supply for almost any element in the periodic table is available with the highest purity. After ion implantation, the damaged crystal can be recrystallised extremely rapidly by pulsed laser melting (PLM), which is a highly non-equilibrium liquid phase epitaxy technique. The combination of these two processes (ion implantation and PLM) has been demonstrated to successfully introduce some element at the concentration up to several orders of magnitude above the solid solubility limit, such as indium in $\mathrm{Si} \mathrm{(200} \mathrm{times),} \mathrm{bismuth} \mathrm{(Bi)} \mathrm{in} \mathrm{Si}$ (500 times) [64] or $\mathrm{Au}$ in Si (by more than a factor of $10^{5}[65]$ ). Therefore, ion implantation followed by pulsed laser melting, i.e. ion beam synthesis, is a potential method for the synthesis of the Ge-Sn alloy.

The first reports on using ion beam synthesis of Ge-Sn were not particularly successful. For example, Bhatia et al. achieved very poor crystallinity with a rough surface and subsurface pores, following PLM of $\mathrm{Sn}$ implanted $\mathrm{Ge}$ at room temperature to high dose $\left(3 \cdot 10^{16} \mathrm{~cm}^{-2}\right.$ at $\left.150 \mathrm{keV}\right)$ [52]. Gao et al. [53] achieved slightly better results, with improved but still poor crystal quality showing well-ordered crystal columns for a $\mathrm{Sn}$ concentration of $\sim 1 a t$. \%, which is too low for a direct band gap transition. It is probable that porosity in Ge induced by high dose implantation is the reason for the low crystal quality in the first study and the lower-than-expected Sn concentration in the second.

Porosity in Ge caused by heavy ion bombardment is well known and has been a subject of study for many years in the ion beam community. In 1982, Appleton et al. were the first to report unusual surface craters on Ge after implantation with ${ }^{209} \mathrm{Bi}$ at the dose of $4 \cdot 10^{15} \mathrm{~cm}^{-2}$ at room temperature [66]. These craters extended several hundred nanometres below the surface as shown for In implantation in Fig. 7. This finding was later confirmed by numerous reports using many different types of ions, such as $\mathrm{Bi}, \mathrm{Au}, \mathrm{I}, \mathrm{Sb}, \mathrm{Sn}, \mathrm{In}, \mathrm{Ni}, \mathrm{Mn}, \mathrm{Ge}$, and $\mathrm{Ga}$ [67-71]. The implant dose at which the onset of porosity occurs depends on several implant parameters, such as the atomic mass of the implanted ions, the implant energy, the dose rate and especially the temperature of the target. At room temperature, the onset of the porosity occurs at an 


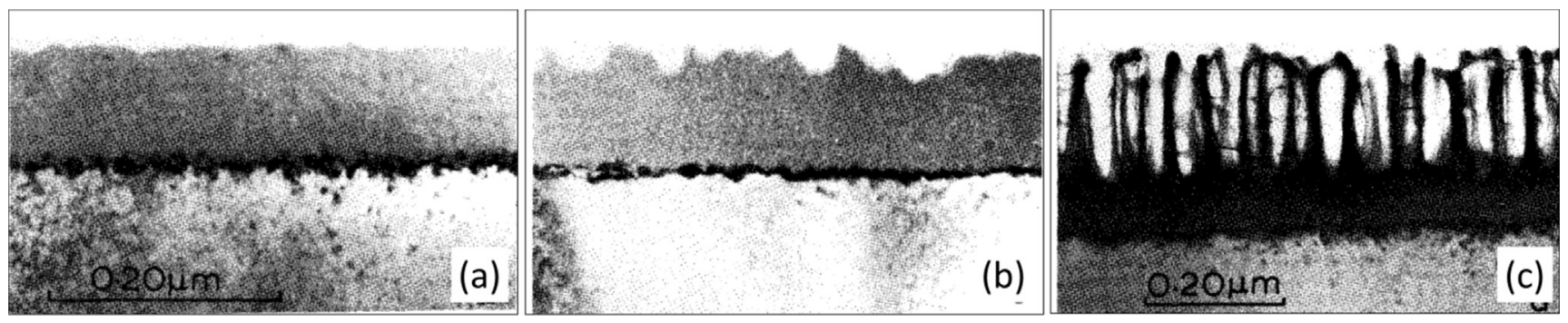

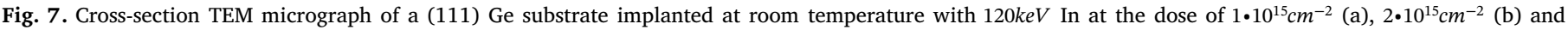
$5 \cdot 10^{15} \mathrm{~cm}^{-2}$ (c) [72].

implant dose of the order of $1 \cdot 10^{15} \mathrm{~cm}^{-2}$ for a keV energy implant. Therefore, it is reasonable to expect that in the previous two Ge-Sn experiments, Ge had become porous after implantation at room temperature because the implant dose in both studies far exceeded $1 \cdot 10^{15} \mathrm{~cm}^{-2}$.

The problem with the porosity is two-fold. Firstly, it is not possible to recover the crystal structure either by liquid phase epitaxy or solid phase epitaxy. Preliminary studies using Q-switched ruby laser annealing as well as thermal annealing showed poor crystal recovery of the porous layer $[66,69,72]$. Secondly, loss of the implanted species due to sputtering that is enhanced by porosity severely limits the concentration implanted into $\mathrm{Ge}$. In particular, as $\mathrm{Ge}$ becomes porous during implantation, the surface area of the sample increases by several orders of magnitude and hence sputtering, which is a surface phenomenon, is much enhanced.

As initially pointed out in the first study by Appleton et al. [54], the porosity effect is temperature-dependent. They found that porosity was totally suppressed for liquid nitrogen $\left(\mathrm{LN}_{2}\right)$ temperature Bi implantation within an implant dose range up to $4 \cdot 10^{15} \mathrm{~cm}^{-2}$ at $280 \mathrm{keV}$. In other studies $[68,71]$, porosity only appeared within the target temperature range of $-80^{\circ} \mathrm{C}-200^{\circ} \mathrm{C}$ for a $\mathrm{Ge}$ implant into Ge up to a dose of $1 \cdot 10^{17} \mathrm{~cm}^{-2}$. Although it is not totally decisive, porosity in Ge is believed to be the consequence of vacancy clustering during implantation once the near-surface becomes amorphous. At elevated temperature, such as $>200^{\circ} \mathrm{C}$, mobile vacancies and interstitials recombine and the crystal structure of $\mathrm{Ge}$ is largely preserved, whereas, at low temperature of $<-80^{\circ} \mathrm{C}$ an amorphous layer occurs and the mobility of vacancies is reduced, hence suppressing the vacancy clustering and the formation of pores. Thus, in the context of implanting Sn into Ge, a first study by Tran et al. used Sn implants at liquid nitrogen temperature to try to supress porosity and demonstrated, followed by pulsed laser melting (PLM), that this was a feasible method for synthesising a high quality Ge-Sn alloy [73].

\subsection{Synthesis of 6 at\%Sn alloy by implanting at low substrate temperature}

In the study by Tran et al. on Sn implanted Ge mentioned above, the starting material was (100) bulk Ge and the Sn ion energy was $100 \mathrm{keV}$, over a dose range of $1.3-2.1 \cdot 10^{16} \mathrm{~cm}^{-2}$. The substrates were kept at $\mathrm{LN}_{2}$ temperature to prevent the formation of porosity. Pulsed laser melting was done with a single pulse of a frequency tripled Nd: YAG laser (wavelength: $355 \mathrm{~nm}$, pulse duration: $9 \mathrm{~ns}$, laser fluence: $0.43 \mathrm{~J} \cdot \mathrm{cm}^{-2}$ and laser spot size: $4 \times 4 \mathrm{~cm}^{2}$ ). This method resulted in a peak $\mathrm{Sn}$ concentration retained in Ge of $\sim 6.6 a t . \%$ after implantation and $\sim 6.2 a t . \%$ after PLM as measured by Rutherford Backscattering Spectrometry (RBS) [73]. Thus, the achieved Sn concentration is improved six-fold as compared to the previous studies and quite close to the required Sn concentration for a direct band gap transition in a fully-relaxed material.

For the above study, RBS and channelling (RBS/C) showed excellent crystal quality of the samples with a calculated substitutionality of $\mathrm{Sn}$ close to $100 \%$, especially for the lower end of the implant dose range. Raman spectroscopy clearly showed the incorporation of $\mathrm{Sn}$ with a monotonic increase of the Ge-Sn phonon mode as a function of the $\mathrm{Sn}$ concentration [61]. Optical characterisation using spectroscopic ellipsometry indicated the lowering of the conduction band at the $\Gamma$ valley from $0.81 \mathrm{eV}$ to $0.77 \mathrm{eV}$, as expected for Ge-Sn alloys, indicating the expected transformation of the materials towards a direct band gap semiconductor [73]. However, as shown by RBS, Raman spectroscopy and X-ray diffraction (XRD) the crystal quality of the samples was not equivalent across the range of the implant doses. The quality appeared to reduce for higher implant doses, again due to the onset of ion-beam induced porosity, as shown below.

Fig. 8 shows the cross-section TEM of the samples implanted with the doses of $1.7 \cdot 10^{16} \mathrm{~cm}^{-2}$ (a,b) and $2.1 \cdot 10^{16} \mathrm{~cm}^{-2}$ (c,d). The lower dose sample after implantation (Fig. 8(a)) had a relatively flat surface and amorphous/crystalline interface. After PLM (Fig. 8(b)), crystal quality of the lower dose sample was excellent as there was no noticeable defects in the layer. However, for the higher dose sample after implantation (Fig. 8(c)) the sample's surface had many large pits which are determined to be the onset of porosity even for the implant at $\mathrm{LN}_{2}$ temperature. This seems to be at odds with studies referred to in the previous section where porosity is totally suppressed for $\mathrm{a}^{74} \mathrm{Ge}$ implant at the doses up to $1 \cdot 10^{17} \mathrm{~cm}^{-2}$ at $\mathrm{LN}_{2}$ temperature [68]. The case for $\mathrm{Sn}$ implantation shows that the phenomenon is also ion mass-dependent. For a ${ }^{120} \mathrm{Sn}$ implant, the onset of porosity is around an implant dose of $2 \cdot 10^{16} \mathrm{~cm}^{-2}$ at $\mathrm{LN}_{2}$ temperature. Although, low substrate temperature can push the onset towards a significantly higher implant dose, it cannot eliminate the phenomenon completely [74]. Additionally, in agreement with the original study of Ge porosity [66] the pores cannot be removed by liquid phase epitaxy as shown in Fig. 8(d). The large pits lead to large defective regions on the surface after PLM, causing reduced crystal quality in samples with higher implant doses as was indicated by RBS, Raman spectroscopy and XRD. However, at lower doses, there may be useful applications of these films since it is also noteworthy that a Sn concentration of just $2 a t$. \% is sufficient for a GeSn alloy to extend the photon absorption to a wavelength of $1750 \mathrm{~nm}$, covering all the bands of the telecommunication windows (from $\mathrm{O}$ to $\mathrm{U}$ ) [49]. Therefore, this technique might be ideal for the fabrication of Ge photodetectors in the near infrared region.

\subsection{Complete suppression of porosity in Ge by a nanometer capping layer of silicon dioxide}

Although low temperature implantation was shown to increase the achieved Sn concentration up to $\sim 6 a t$. $\%$, the onset of porosity has limited the maximum implant dose to around $2 \cdot 10^{16} \mathrm{~cm}^{-2}$. A fully developed ion beam synthesis technique for direct band gap Ge-Sn alloys, however, will need to provide both high quality material and Sn concentrations of $>10$ at. \%. Towards this goal, recently it has been shown that a nanometer capping layer of $\mathrm{SiO}_{2}$ can effectively suppress the formation of porosity during implantation up to the doses of around $4.5 \cdot 10^{16} \mathrm{~cm}^{-2}$ for a ${ }^{120} \mathrm{Sn}$ implant at the energy of $120 \mathrm{keV}[75,76]$. Fig. 9 shows the Sn concentration after implantation, as measured by RBS, as a function of implant dose for the Ge samples without a cap and samples with a cap of $40 \mathrm{~nm} \mathrm{SiO}_{2}$. The maximum $\mathrm{Sn}$ concentration in the 

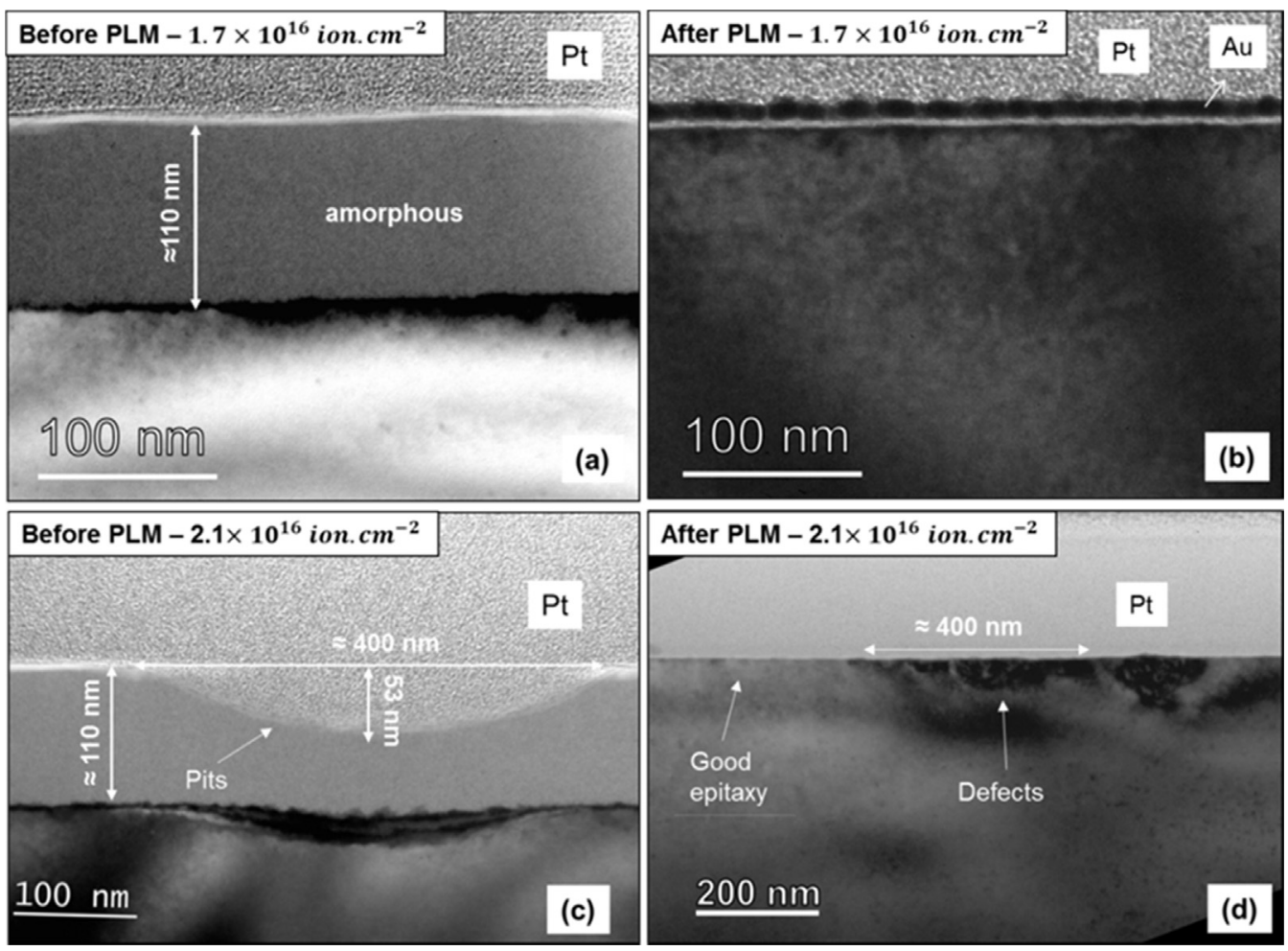

Fig. 8. TEM micrographs of the $1.7 \cdot 10^{16} \mathrm{~cm}^{-2}$ sample after implantation (a), after PLM (b) and of the $2.1 \cdot 10^{16} \mathrm{~cm}^{-2}$ sample after implantation (c) and after PLM (d) [73]

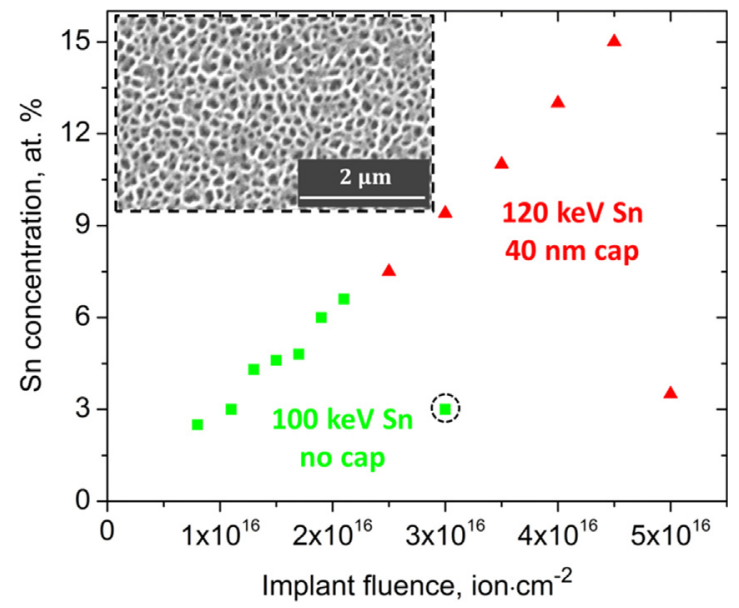

Fig. 9. The peak Sn concentration as a function of implant dose at an implant energy of $100 \mathrm{keV}$ without a cap (square-green) and at $120 \mathrm{keV}$ with a cap (triangle-red) [75]. Inset is a SEM micrograph of the $3 \cdot 10^{16} \mathrm{~cm}^{-2}$ sample without a cap.

uncapped sample is 6.6 at. $\%$ at the dose of $\sim 2 \cdot 10^{16} \mathrm{~cm}^{-2}$, and at an implant dose of $3 \cdot 10^{16} \mathrm{~cm}^{-2}$, the sample is decidedly porous as shown by the inset of Fig. 9. Porosity-enhanced sputtering also causes the Sn concentration to be limited to $\sim 3 a t$. \%. On the other hand, samples with a capping layer show no porosity and a remarkable increase of the available $\mathrm{Sn}$ concentration. For example, at a dose of $4.5 \cdot 10^{16} \mathrm{~cm}^{-2}$, the measured Sn concentration is $\sim 15 a t$. \%. There were no sign of surface porosity in all samples with a cap until a dose of $5 \cdot 10^{16} \mathrm{~cm}^{-2}$, when the cap was sputtered off and the surface rendered porous.

Thus, using a capping layer of $\mathrm{SiO}_{2}$ during implantation was possible to prevent the surface from the deleterious porosity. After removing the remaining $\mathrm{SiO}_{2}$ layer by dipping the samples into hydrofluoric acid, PLM was able to regrow the Ge-Sn layer as shown in Fig. 10 [77]. The cross-section TEM image of the $3 \cdot 10^{16} \mathrm{~cm}^{-2}$ sample indicated

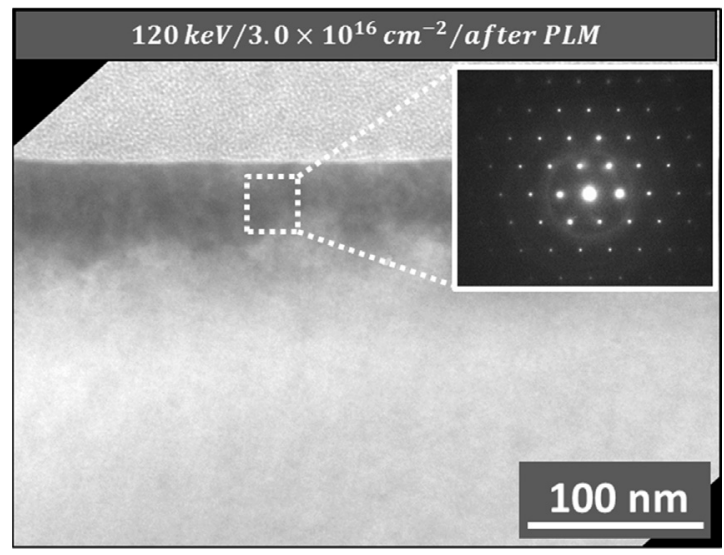

Fig. 10. Cross-section TEM of the $3 \cdot 10^{16} \mathrm{~cm}^{-2}$ sample after PLM. Inset is the electron diffraction pattern of the Ge-Sn layer [77].

that the Ge-Sn layer has high crystal quality. Electron diffraction (inset) from the layer shows a typical diamond cubic structure. Characterisation using RBS determined the Sn concentration of the material to be 9 at. \% with a Sn substitutionality of $85-90 \%$. The slightly reduced substitutional percentage of $\mathrm{Sn}$ as compared to the previous result is believed to be a result of the large compressive strain built up in the material at high $\mathrm{Sn}$ concentration, due to the lattice mismatch between the Ge substrate and the GeSn layer. XRD-reciprocal space mapping indicated the thin film of $9 a t . \% S n$ was fully compressively strained [77]. The increasing compressive strain with the Sn concentration will eventually lead to poor crystal growth by liquid phase epitaxy and/or Sn precipitation. At this point, the limit to the achievable substitutional Sn concentration is not so much the low solid solubility in Ge of $\sim 0.5 a t . \%$, but the build-up of compressive strain in the material, as discussed further below.

A particular undesirable consequence of compressive strain is the role it plays in affecting the direct band gap transition of the material, 


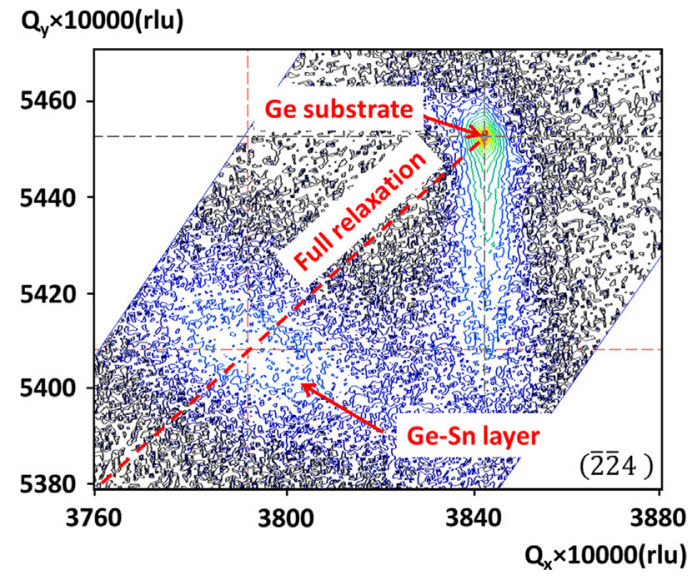

Fig. 11. Reciprocal space mapping on the $(\overline{2} \overline{2} 4)$ planes of the $6.0 \cdot 10^{16} \mathrm{~cm}^{-2}$ sample.

as shown in Fig. 3, where compressive strain increases the $\mathrm{Sn}$ concentration required for a direct band gap transition. Higher Sn concentration will in turn induce more compressive strain into the material. Finding an effective way to relax the material without introducing defects should be of prime concerns in Ge-Sn research. We note that, according to Ref. [29], if the Ge-Sn layer is grown pseudo-morphically on a Ge substrate $\left(a_{G e-S n \|}=a_{G e \|}\right)$, there is no Sn concentration sufficient for a direct band gap transition, because the reduced band gap difference between the $\Gamma$ and the $L$ valleys, due to the $S n$ incorporation, is offset by the increased compressive strain.

\subsection{Strain-relaxed Ge-Sn alloys}

Recently, progress in producing a highly-relaxed Ge-Sn alloy using ion implantation followed by PLM has been demonstrated. The principle is to produce a thicker film by implanting at a higher energy. In this case, $2 \mu \mathrm{m}$ Ge-on-Si substrates were implanted with ${ }^{120} \mathrm{Sn}$ ions at the energy of $350 \mathrm{keV}$ and at a dose of $4-6.8 \cdot 10^{16} \mathrm{~cm}^{-2}$. The substrates were deposited with $40 \mathrm{~nm} \mathrm{SiO}_{2}$ and kept at $\mathrm{LN}_{2}$ temperature during implantation. RBS measurements showed the thickness of the amorphous layer after implantation was $360 \mathrm{~nm}$ and the $\mathrm{Sn}$ concentration was $\sim 8.5$ at. \% for the $6 \cdot 10^{16} \mathrm{~cm}^{-2}$ implant. After PLM, the Sn concentration was slightly reduced to $\sim 7.5 a t$. $\%$ and the substitutionality was $\sim 82 \%$.

Strain characteristic of the thick Ge-Sn layer was studied by XRDreciprocal space mapping (RSM). Fig. 11 is the RSM scan along the asymmetric crystal direction $[\overline{2} \overline{2} 4]$ of the $6 \cdot 10^{16} \mathrm{~cm}^{-2}$ sample. $Q_{\mathrm{x}}$ and $\mathrm{Q}_{\mathrm{y}}$ are the reciprocal lattice units along the direction parallel and normal to the surface, respectively. It is clear in the RSM map that the Ge-Sn layer is fully-relaxed as the signal from the layer lies on the full relaxation axis. The calculated lattice constant of the Ge-Sn layer is $\sim 5.7 \AA$, whereas the lattice constant of the pristine $\mathrm{Ge}$ is $\sim 5.65 \AA$. Lattice expansion of the Ge-Sn layer as compared to the pristine Ge is calculated to be $0.9 \%$. Considering the Sn concentration of $6.5 a t$. \% is sufficient for a relaxed Ge-Sn alloy to be a direct band gap material, photoluminescence (PL) was conducted on a series of the relaxed samples. The preliminary PL result showed considerable signal from the samples. However, the intensity of the PL was not consistent with the Sn concentration. Further work and process innovation are necessary to improve the quality of the relaxed Ge-Sn alloys so that they are suitable in practical electronic and photonic applications.

\section{Conclusion}

Although CVD is still the preferred method preparing Ge-Sn alloys, there remain significant issues and limitations, which are affecting the drive towards a room temperature mid-infrared laser. Alternatively, much of the groundwork has been done to demonstrate that ion implantation followed by pulsed laser melting can be a competitive method to CVD to produce a direct band gap Ge-Sn alloy with high crystal quality. Inherently, the technique is very reproducible, provides high throughput as well as virtually no undesired impurity issues. It is now demonstrated to be capable of realising a Sn concentration of at least 10 at. \%. A pathway for the complete strain relaxation, which is very important for the material, is also presented. We believe an intrinsically direct band gap Ge-Sn alloy with excellent crystal quality is within reach in the near future.

\section{References}

[1] H. Park, A.W. Fang, S. Kodama, J.E. Bowers, Opt. Express 13 (2005) 9460.

[2] A.W. Fang, H. Park, O. Cohen, R. Jones, M.J. Paniccia, J.E. Bowers, Opt. Express 14 (2006) 9203.

[3] R. Chen, T.-T.D. Tran, K.W. Ng, W.S. Ko, L.C. Chuang, F.G. Sedgwick, C. ChangHasnain, Nat. Photonics 5 (2011) 170.

[4] H. Liu, T. Wang, Q. Jiang, R. Hogg, F. Tutu, F. Pozzi, A. Seeds, Nat. Photonics 5 (2011) 416.

[5] R. Claps, D. Dimitropoulos, Y. Han, B. Jalali, Opt. Express 10 (2002) 1305.

[6] O. Boyraz, B. Jalali, Opt. Express 12 (2004) 5269.

[7] H. Rong, A. Liu, R. Jones, O. Cohen, D. Hak, R. Nicolaescu, A. Fang, M. Paniccia, Nature 433 (2005) 292.

[8] H. Rong, R. Jones, A. Liu, O. Cohen, D. Hak, A. Fang, M. Paniccia, Nature 433 (2005) 725 .

[9] R. Soref, IEEE J. Sel. Top. Quantum Electron. 12 (2006) 1678.

[10] A. Polman, J. Appl. Phys. 82 (1997) 1.

[11] T.J. Kippenberg, J. Kalkman, A. Polman, K.J. Vahala, Phys. Rev. A 74 (2006) 051802.

[12] C. Angulo Barrios, M. Lipson, Opt. Express 13 (2005) 10092.

[13] M.S. Shur, Handbook series on semiconductor parameters 1 World Scientific, 1996.

[14] M. Pairot, I. Zoran, H. Paul, Semicond. Sci. Technol. 22 (2007) 742.

[15] J.R. Chelikowsky, M.L. Cohen, Phys. Rev. B 14 (1976) 556.

[16] Y.M. Niquet, D. Rideau, C. Tavernier, H. Jaouen, X. Blase, Phys. Rev. B 79 (2009) 245201

[17] F. Zhang, V.H. Crespi, P. Zhang, Phys. Rev. Lett. 102 (2009) 156401.

[18] P. Vogl, M.R. Martin, J.A. Majewski, G. Abstreiter, Phys. Scr. 1993 (() (1993) 476.

[19] J. Liu, X. Sun, D. Pan, X. Wang, L.C. Kimerling, T.L. Koch, J. Michel, Opt. Express 15 (2007) 11272.

[20] J. Liu, X. Sun, L.C. Kimerling, J. Michel, Opt. Lett. 34 (2009) 1738.

[21] G.A. Slack, S.F. Bartram, J. Appl. Phys. 46 (1975) 89.

[22] X. Sun, J. Liu, L.C. Kimerling, J. Michel, Opt. Lett. 34 (2009) 1198.

[23] J. Liu, X. Sun, R. Camacho-Aguilera, L.C. Kimerling, J. Michel, Opt. Lett. 35 (2010) 679.

[24] R.E. Camacho-Aguilera, Y. Cai, N. Patel, J.T. Bessette, M. Romagnoli, L.C. Kimerling, J. Michel, Opt. Express 20 (2012) 11316.

[25] S. Fischler, J. Appl. Phys. 33 (1962) 1615.

[26] M.J. Suess, et al., Nat. Photon 7 (2013) 466.

[27] J.R. Sánchez-Pérez, C. Boztug, F. Chen, F.F. Sudradjat, D.M. Paskiewicz, R. Jacobson, M.G. Lagally, R. Paiella, Proceedings of the National Academy of Sciences 108, 18893, 2011.

[28] J.R. Jain, A. Hryciw, T.M. Baer, D.A.B. Miller, M.L. Brongersma, R.T. Howe, Nat. Photon 6 (2012) 398

[29] S. Gupta, B. Magyari-Köpe, Y. Nishi, K.C. Saraswat, J. Appl. Phys. 113 (2013) 073707.

[30] D.W. Jenkins, J.D. Dow, Phys. Rev. B 36 (1987) 7994.

[31] K.A. Mäder, A. Baldereschi, H. von Känel, Solid State Commun. 69 (1989) 1123.

[32] W.-J. Yin, X.-G. Gong, S.-H. Wei, Phys. Rev. B 78 (2008) 161203.

[33] V.R. D'Costa, C.S. Cook, A.G. Birdwell, C.L. Littler, M. Canonico, S. Zollner, J. Kouvetakis, J. Menéndez, Phys. Rev. B 73 (2006) 125207.

[34] R.W. Olesinski, G.J. Abbaschian, Bulletin of Alloy Phase Diagrams 5, 265.

[35] S. Oguz, W. Paul, T.F. Deutsch, B.Y. Tsaur, D.V. Murphy, Appl. Phys. Lett. 43 (1983) 848.

[36] F. Hirofumi, M. Hiroaki, N. Toshio, I. Takeshi, O. Yukio, Jpn. J. Appl. Phys. 25 (1986) 1312.

[37] I. Chambouleyron, F.C. Marques, J.P. d. Souza, I.J.R. Baumvol, J. Appl. Phys. 63 (1988) 5596.

[38] I.T.H. Chang, B. Cantor, A.G. Cullis, J. Non-Cryst. Solids 117-118 (1990) 263.

[39] W. Wegscheider, J. Olajos, U. Menczigar, W. Dondl, G. Abstreiter, J. Cryst. Growth 123 (1992) 75

[40] A. Harwit, P.R. Pukite, J. Angilello, S.S. Iyer, Thin Solid Films 184 (1990) 395.

[41] G. He, H.A. Atwater, Appl. Phys. Lett. 68 (1996) 664.

[42] R. Chen, H. Lin, Y. Huo, C. Hitzman, T.I. Kamins, J.S. Harris, Appl. Phys. Lett. 99 (2011) 181125.

[43] M. Rojas-López, H. Navarro-Contreras, P. Desjardins, O. Gurdal, N. Taylor, J.R.A. Carlsson, J.E. Greene, J. Appl. Phys. 84 (1998) 2219.

[44] J. Werner, M. Oehme, M. Schmid, M. Kaschel, A. Schirmer, E. Kasper, J. Schulze, Appl. Phys. Lett. 98 (2011) 061108.

[45] N. Bhargava, M. Coppinger, J. Prakash Gupta, L. Wielunski, J. Kolodzey, Appl. Phys. Lett. 103 (2013).

[46] J. Taraci, J. Tolle, J. Kouvetakis, M.R. McCartney, D.J. Smith, J. Menendez, 
M.A. Santana, Appl. Phys. Lett. 78 (2001) 3607.

[47] M. Bauer, J. Taraci, J. Tolle, A.V.G. Chizmeshya, S. Zollner, D.J. Smith, J. Menendez, C. Hu, J. Kouvetakis, Appl. Phys. Lett. 81 (2002) 2992.

[48] M.R. Bauer, C.S. Cook, P. Aella, J. Tolle, J. Kouvetakis, P.A. Crozier, A.V.G. Chizmeshya, D.J. Smith, S. Zollner, Appl. Phys. Lett. 83 (2003) 3489.

[49] J. Mathews, R. Roucka, J. Xie, S.-Q. Yu, J. Menéndez, J. Kouvetakis, Appl. Phys. Lett. 95 (2009) 133506.

[50] R. Roucka, J. Mathews, C. Weng, R. Beeler, J. Tolle, J. Menendez, J. Kouvetakis, IEEE J. Quantum Electron. 47 (2011) 213.

[51] C.L. Senaratne, P.M. Wallace, J.D. Gallagher, P.E. Sims, J. Kouvetakis, J. Menéndez, J. Appl. Phys. 120 (2016) 025701.

[52] J. Mathews, R.T. Beeler, J. Tolle, C. Xu, R. Roucka, J. Kouvetakis, J. Menéndez, Appl. Phys. Lett. 97 (2010) 221912.

[53] R. Roucka, J. Mathews, R.T. Beeler, J. Tolle, J. Kouvetakis, J. Menéndez, Appl. Phys. Lett. 98 (2011) 061109.

[54] B. Vincent, et al., Appl. Phys. Lett. 99 (2011) 152103.

[55] R. Chen, Y.-C. Huang, S. Gupta, A.C. Lin, E. Sanchez, Y. Kim, K.C. Saraswat, T.I. Kamins, J.S. Harris, J. Cryst. Growth 365 (2013) 29.

[56] A. Mosleh, S.A. Ghetmiri, B.R. Conley, M. Hawkridge, M. Benamara, A. Nazzal, J. Tolle, S.-Q. Yu, H.A. Naseem, J. Elec Mater. 43 (2014) 938.

[57] S. Wirths, D. Buca, G. Mussler, A. Tiedemann, B. Holländer, P. Bernardy, T. Stoica, D. Grützmacher, S. Mantl, ECS J. Solid State Sci. Technol. 2 (2013) N99.

[58] S. Wirths, et al., Nat. Photon 9 (2015) 88.

[59] S. Al-Kabi, et al., Appl. Phys. Lett. 109 (2016) 171105.

[60] J. margetis, et al., ACS Photonics (2017).

[61] R.F. Spohn, C.B. Richenberg, ECS Trans. 50 (2013) 921.

[62] Voltaix, Material Safety Data Sheet for: Flammable Stannane-d4 (SnD4) mix, 2009.
[63] R.F. Spohn, A. Sinha, C.B. Richenberg, (Google Patents, 2013.

[64] C.W. White, B.R. Appleton, S.R. Wilson, J.M.P.W. Mayer (Ed.), Laser Annealing of Semiconductors, Academic Press, 1982, p. 111.

[65] W. Yang, et al., Phys. Rev. Mater. 1 (2017) 074602.

[66] B.R. Appleton, O.W. Holland, J. Narayan, O.E. Schow Iii, J.S. Williams, K.T. Short, E. Lawson, Appl. Phys. Lett. 41 (1982) 711.

[67] I.H. Wilson, J. Appl. Phys. 53 (1982) 1698.

[68] B. Stritzker, R.G. Elliman, J. Zou, Nucl. Instrum. Methods Phys. Res. Sect. B: Beam Interact. Mater. At. 175-177 (2001) 193.

[69] T. Janssens, C. Huyghebaert, D. Vanhaeren, G. Winderickx, A. Satta, M. Meuris, W. Vandervorst, J. Vac. Sci. Technol. B 24 (2006) 510.

[70] L. Romano, G. Impellizzeri, M.V. Tomasello, F. Giannazzo, C. Spinella, M.G. Grimaldi, J. Appl. Phys. 107 (2010) 084314.

[71] H.S. Alkhaldi, F. Kremer, T. Bierschenk, J.L. Hansen, A. Nylandsted-Larsen, J.S. Williams, M.C. Ridgway, J. Appl. Phys. 119 (2016) 094303.

[72] O.W. Holland, B.R. Appleton, J. Narayan, J. Appl. Phys. 54 (1983) 2295.

[73] T.T. Tran, D. Pastor, H.H. Gandhi, L.A. Smillie, A.J. Akey, M.J. Aziz, J.S. Williams, J. Appl. Phys. 119 (2016) 183102.

[74] E. Bruno, G.G. Scapellato, G. Bisognin, E. Carria, L. Romano, A. Carnera, F. Priolo, J. Appl. Phys. 108 (2010) 124902.

[75] T.T. Tran, H.S. Alkhaldi, H.H. Gandhi, D. Pastor, L.Q. Huston, J. Wong-Leung, M.J. Aziz, J.S. Williams, Appl. Phys. Lett. 109 (2016) 082106.

[76] H.S. Alkhaldi, T.T. Tran, F. Kremer, J.S. Williams, J. Appl. Phys. 120 (2016) 215706.

[77] T.T. Tran, H.H. Gandhi, D. Pastor, M.J. Aziz, J.S. Williams, Materials Science in Semiconductor Processing. 